Accepted for Publication in the Astronomical Journal

\title{
THE SPECTACULAR IONIZED INTERSTELLAR MEDIUM OF NGC 55
}

\author{
Annette M. N. Ferguson ${ }^{1}$ and Rosemary F. G. Wyse $^{1}$ \\ Department of Physics and Astronomy, The Johns Hopkins University, \\ Baltimore, MD 21218 \\ J. S. Gallagher \\ Department of Astronomy, University of Wisconsin, Madison, WI 53706
}

\begin{abstract}
We present deep $\mathrm{H} \alpha+[\mathrm{NII}]$, [SII] $(\lambda \lambda 6716,6731 \AA)$ and [OII] $(\lambda \lambda 3726,3729$ $\AA)$ images of the highly inclined, actively star-forming SBm galaxy NGC 55, located in the nearby Sculptor Group. Due to its proximity, NGC 55 provides a unique opportunity to study the disk-halo interface in a late-type galaxy with unprecedented spatial resolution. Our images reveal a spectacular variety of ionized gas features, ranging from giant HII region complexes, to supergiant filamentary and shell features, to patches of very faint diffuse emission. Many of these features protrude well above the plane of the galaxy, including a very faint fragmented shell of emission which is visible at $2.6 \mathrm{kpc}$ above the disk. We identify candidate 'chimneys' extending out of the disk, which could be the conduits into the halo for hot gas around disk star-forming regions, and could also provide low-density paths for the passage of UV photons from the disk to the halo. Several of the identified chimneys are 'capped' with clumps of ionized gas, one of which, located at $1.5 \mathrm{kpc}$ above the disk plane, appears to be the site of recent star formation. Emission-line ratios ([OII]/H $\alpha+[\mathrm{NII}],[\mathrm{SII}] / \mathrm{H} \alpha+[\mathrm{NII}])$ constrain the ionization mechanism of the gas, and our images allow the first measurement of $[\mathrm{OII}] / \mathrm{H} \alpha+[\mathrm{NII}]$ in extra-planar diffuse ionized gas. The diffuse gas is characterized by emission-line ratios which are enhanced on average by a factor of two compared to those of bright HII regions. Each line ratio increases in value smoothly from the cores of HII regions, through the haloes of HII
\end{abstract}

\footnotetext{
${ }^{1}$ Visiting Astronomer, Cerro Tololo InterAmerican Observatory. CTIO is operated by AURA, Inc. under contract to the National Science Foundation.
} 
regions, into the diffuse ionized gas. Such a continuous trend is predicted by models in which the diffuse gas is ionized by photons produced by massive stars in HII regions. We discuss the factors which may determine the existence of extra-planar ionized gas in galaxies.

Subject headings: Galaxies: Individual (NGC 55) - Galaxies: Irregular Galaxies: ISM - ISM: bubbles - ISM: HII regions - ISM: structure 


\section{Introduction}

The interstellar disk-halo connection plays an important role in understanding the processes of disk galaxy evolution (e.g. Bloemen 1991). Many theories have been proposed by which star formation in the disk causes continuous and/or sporadic transfer of mass and energy from the disk to the halo, with the possible return of halo material to the disk (e.g. Shapiro \& Field 1976, Norman \& Ikeuchi 1989; hereafter NI89). Such a cycle could regulate the star formation process in disks, leading to low overall star-formation efficiency, as well as provide a means by which metal-enriched gas could be distributed over large regions of galaxies. This disk-halo interaction may also help in creating and sustaining the ionized gaseous haloes inferred to exist around galaxies from quasar absorption-line studies (e.g. Bergeron \& Boisse 1991).

Several pieces of evidence suggest a strong coupling between the disk and halo in galaxies. In the Milky Way, structures resembling 'worms' in the atomic gas have been found, typically extending several hundred parsecs out of the disk into the halo (e.g. Heiles 1984; Normandeau, Taylor \& Dewdney 1996) and there exists a thick ionized gas layer, the 'Reynolds layer' with a scale-height of $\sim 1$ kpc (Reynolds et al 1971; Reynolds 1984). Maintaining the ionization of this layer requires a large power that can be met easily only by ionizing photons from OB stars (e.g. Reynolds 1984; Kulkarni and Heiles 1988) however these photons must be able to travel from their point of origin in the disk to significant heights above the plane. Other possibilities for the origin of the ionizing photons have been proposed and include shocks, turbulent mixing layers (Slavin, Shull \& Begelman 1993) and galactic microflares (Raymond 1992). In recent years, deep narrow-band imaging of highly inclined external disk galaxies has revealed that some form of diffuse ionized gas, a term commonly used to refer to ionized gas lying outside the boundaries of traditional HII regions, is quite often found above the disk plane (e.g. Rand 1996). Most often, the extra-planar ionized gas is seen in the form of discrete structures, such as loops, filaments and plumes, occasionally accompanied by an apparently unstructured, thick diffuse layer (e.g. Rand, Kulkarni \& Hester 1990, 1992; Pildis, Bregman \& Schombert 1994; Rand 1996). Diffuse ionized gas has also been observed in several face-on galaxies (e.g. Monnet 1971; Hunter \& Gallagher 1990, 1997; Walterbos \& Braun 1994; Ferguson et al 1996), where a clear correlation with sites of very recent star formation has emerged.

Local energy and momentum input, and local mass surface density, govern the disk-halo connection (e.g. NI89, Heiles 1990). Only the most luminous OB associations are likely to produce superbubbles which can 'break-out' of the disk, and thus relatively late-type systems, which typically host the most luminous HII regions (Kennicutt 1988), are of extreme interest. Low mass, low luminosity galaxies, such as the LMC are observed 
to host several supershells and giant filaments (e.g. Davies, Elliot \& Meaburn 1976, Hunter, Hawley \& Gallagher 1993) but the vertical extent of these structures is unknown.

We here present a study of the disk-halo interface in the nearby highly inclined SBm galaxy NGC 55, located in the nearby Sculptor Group. Our study is based on large field-of-view $\mathrm{H} \alpha+[\mathrm{NII}]$, [SII] $(\lambda \lambda 6717,6731 \AA)$ and [OII] $(\lambda \lambda 3727,3729 \AA)$ images. Due to its proximity, NGC 55 provides a unique opportunity to study the disk-halo interface in a late-type galaxy with unprecedented spatial resolution. The basic properties of the galaxy are listed in Table 1 . Hoopes et al (1996) have recently analysed $\mathrm{H} \alpha+[\mathrm{NII}]$ and [SII] images of NGC 55, taken with the CTIO Schmidt telescope. The data presented here are of considerably higher spatial resolution and sensitivity, and include images in the important [OII] emission line, allowing a more detailed study of structure and ionization state of the gas in the disk-halo interface.

\section{Observations and Data Reduction}

Images of NGC 55 were obtained using the CTIO $1.5 \mathrm{~m}+$ Tek 2048 x 2048 CCD during September 1994 and 1995. At f/7.5, the pixel scale was $0.44^{\prime \prime}$, corresponding to physical size of $\sim 4$ pc at the distance adopted here for NGC 55 of $1.6 \mathrm{Mpc}$. The filters used

for the observations were well-matched to the galaxy's recessional velocity and consisted of the following: (i) $68 \AA$ (FWHM) H $\alpha$ filter (which also encompassed the [NII] $6548,6584 \AA$ ) lines) and a broadband $\mathrm{R}$ filter for the continuum observations, (ii) $36 \AA$ (FWHM) [SII] filter and a narrow ( $80 \AA$ ) off-band filter for the continuum observations, and (iii) $24 \AA$ (FWHM) [OII] filter and a narrow (130 $\AA$ ) off-band filter for the continuum observations. The field of view was $\sim 15 \times 15 \operatorname{arcmin}^{2}$ (corresponding to $7 \mathrm{kpc}$ on a side at the distance of NGC 55) for the $\mathrm{H} \alpha+[\mathrm{NII}]$ observations and slightly smaller than this for the [OII] and [SII] filters, due to vignetting. Complete spatial coverage of the galaxy was achieved in $\mathrm{H} \alpha$ by three separate pointings; the $[\mathrm{SII}]$ and $[\mathrm{OII}]$ observations were restricted to the central $5 \mathrm{kpc}$. Total exposure times were approximately 2 hours in $\mathrm{H} \alpha$ (per field) and 1.5 hours each in $[\mathrm{SII}]$ and $[\mathrm{OII}]$. The seeing was in the range of $1.5-2.0{ }^{\prime \prime}$.

Reductions were carried out using standard procedures. Well-exposed, median-filtered twilight sky frames were used to flat-field the images, and an accuracy of better than $1 \%$ was typically achieved. Multiple pointings at the approximately the same position were registered using field stars in the frame and those taken through the same filter were combined with an average sigma-clipping technique. The sky value was determined in each image as the mean of the median pixel value in a series of small $100 \times 100$ pixel boxes placed well outside the galaxy, and subtracted off. Foreground stars in the frames were used to 
determine the scaling factors between the emission-line and the continuum images. The scaled continuum images were subsequently subtracted to produce net emission-line images.

Observations of standard stars from the list of Stone and Baldwin (1983) were used to calibrate the $\mathrm{H} \alpha+[\mathrm{NII}]$ and $[\mathrm{SII}]$ images. Our NGC $55 \mathrm{H} \alpha$ frames were largely obtained under non-photometric conditions; the few frames taken under photometric conditions were calibrated using standard stars, then those frames were used to calibrate our final combined images. The [SII] images were obtained under photometric conditions. The [OII] image was calibrated relative to the $\mathrm{H} \alpha$ frame using published spectrophotometric data for several HII regions in the central region of the galaxy (Webster \& Smith 1983). The average sensitivity of the $\mathrm{H} \alpha+[\mathrm{NII}]$ continuum-subtracted image, taken to be $1 \sigma$ of the sky background, is $1.59 \times 10^{-17}$ erg $\mathrm{s}^{-1} \mathrm{~cm}^{-2} \operatorname{arcsec}^{-2}$, corresponding to an emission measure 1 per pixel of $7.8 \mathrm{pc} \mathrm{cm}^{-6}$ (for an assumed electron temperature of $10^{4} \mathrm{~K}$ ). When smoothed with a gaussian of FWHM $=2.5^{\prime \prime}$, to enhance the detection of very faint, extended features, the sky noise decreases to $\mathrm{EM} \sim 1 \mathrm{pc} \mathrm{cm}{ }^{-6}$. The average sensitivities of the unsmoothed [OII] and [SII] images are $4.82 \times 10^{-17} \mathrm{erg} \mathrm{s}^{-1} \mathrm{~cm}^{-2} \operatorname{arcsec}^{-2}$ and $1.32 \times 10^{-17} \mathrm{erg} \mathrm{s}^{-1} \mathrm{~cm}^{-2} \operatorname{arcsec}^{-2}$ respectively. The contribution of $[\mathrm{NII}]$ to our $\mathrm{H} \alpha+[\mathrm{NII}]$ images is expected to be small, based on the published spectra of HII regions from Webster \& Smith (1983). Galactic extinction is negligible in the direction of NGC 55 and no corrections have been applied.

Scattered light is an important issue in studies of faint extended emission, and can be caused either by the telescope optics/detector or, in the particular case of ionized gas, by dust in and around HII regions. Scattering in the optics/detector of the system can be constrained by measuring the azimuthally averaged radial light profile around several bright stars in our fields. From this analysis, we find that $95 \%$ of the total light from a star is contained within a radius of $\sim 6^{\prime \prime}$ in the $\mathrm{H} \alpha+[\mathrm{NII}]$ image, and within a radius of $\sim 5^{\prime \prime}$ in the $[\mathrm{SII}]$ and $[\mathrm{OII}]$ images. Furthermore, in all three images, more than $99 \%$ of the total light from a star is enclosed within a radius of $\sim 20^{\prime \prime}$. Thus, if the faint diffuse ionized gas emission present in NGC 55 were simply a result of scattered light in the telescope, then it should be significantly more localised around HII regions than is observed, and should contribute no more than a few percent of the total emission-line flux measured in each filter. Scattering from dust in the HII regions themselves is unlikely given the distinct morphology of the diffuse emission, as well as the different emission-line ratios of the diffuse emission relative to HII regions.

\footnotetext{
${ }^{1}$ Emission measure is related to Rayleighs, the commonly used unit of surface brightness in Galactic DIG studies, by $\operatorname{EM}\left(\mathrm{pc} \mathrm{cm}^{-6}\right)=2.78 \times \mathrm{I}_{H \alpha}$ (Rayleighs) for $\mathrm{T}_{e}=10^{4} \mathrm{~K}$.
} 


\section{Morphology of the Ionized Gas}

Figure 1 (Plate $\mathrm{X}$ ) shows our $\mathrm{H} \alpha+[\mathrm{NII}]$ continuum-subtracted mosaiced image of the entire galaxy. A spectacular variety of ionized gas features are visible, ranging from bright HII complexes, to loops and plumes protruding out the plane of the galaxy, to faint diffuse emission lying both above, below and within the disk. These features are detected in all three bands, however filamentary features are less pronounced in the [OII] and [SII] images due their lower $\mathrm{S} / \mathrm{N}$. The complex structure seen in the extra-planar ionized gas is striking. Our images underscore the importance of high spatial resolution in studying the structure of the disk-halo interface in galaxies; pending HST observations of NGC 891 (Dettmar, private communication), in which the extra-planar gas studied thus far does not show such detailed structure, will probe a similar spatial scale as the NGC 55 observations presented here.

The most prominent features are the two large HII complexes located in the central regions of NGC 55, which are clearly the dominant sites of recent massive star formation. The $\mathrm{H} \alpha+[\mathrm{NII}]$ luminosities of these features are estimated to be 9 and $3 \times 10^{39} \mathrm{erg} \mathrm{s}^{-1}$ (uncorrected for extinction) and they each span more than a kpc in diameter. Their individual luminosities are comparable to that of the 30 Dor complex in the LMC (Kennicutt et al 1995) and are more slightly luminous than NGC 604, the brightest HII complex in M33 (Kennicutt 1988). The combined luminosity of these two complexes accounts for more than half of the total $\mathrm{H} \alpha+[\mathrm{NII}]$ luminosity of NGC 55, and exceeds that observed for the entire ensemble of HII regions in the disk of M31 (Walterbos \& Braun 1994).

These two HII complexes are surrounded by a wealth of structure. Figure 2 a (Plate XX) shows an $\mathrm{H} \alpha+[\mathrm{NII}]$ image of the central $\sim 5 \mathrm{kpc}$ of the galaxy, with many of these features marked; Table 2 lists their measured properties. Several of the brighter features have been previously discussed: Graham \& Lawrie (1982) identified feature 'D', during a photographic plate search for planetary nebulae; Bomans \& Grebel (1993) identified features ' $\mathrm{F}$ ' and ' $\mathrm{H}$ ' from a small field of view $\mathrm{CCD} \mathrm{H} \alpha$ image centered on the two brightest HII regions. Hoopes et al. (1996) have discussed the general morphology of the ionized gas emission from shallower, lower resolution images obtained with the CTIO Schmidt Telescope.

Several of the ionized gas features in NGC 55 extend considerable distances above the disk plane. Features 'B', 'E', 'G', 'I' and 'K' may be examples of chimney structures, through which hot gas is theorised to be vented into the halo. Such structures are predicted to arise as a result of correlated Type II SNe explosions in OB associations, leading to the production of an expanding superbubble which can break through the disk (e.g. MacLow, McCray \& Norman 1988; Norman \& Ikeuchi 1989; Heiles 1990). In our images, several 
of these features ('G', 'I' and ' $\mathrm{K}$ ') appear to be capped with clumps of relatively bright emission, possibly from gas swept up by the expanding bubble and now directly ionized by the OB association located in the disk below (see Figure 2b). The cap which sits atop feature ' $\mathrm{I}$ ' is particularly interesting, since it is strong in the [OII]-continuum image and may actually be due to in situ star formation at $1.5 \mathrm{kpc}$ above the plane of the disk. It has an $\mathrm{H} \alpha+[\mathrm{NII}]$ luminosity of $\sim 3 \times 10^{36} \mathrm{erg} \mathrm{s}^{-1}$, slightly less luminous than that of the Orion nebula (Kennicutt 1984). On the North side of the galaxy, we have detected several patches of extremely faint $\mathrm{H} \alpha$ emission $\left(\mathrm{EM}<5 \mathrm{pc} \mathrm{cm}^{-6}\right)$ lying at distances up to $2.6 \mathrm{kpc}$ above the plane. These patches, most clearly seen on Figure 2 b (Plate XXX), which has been smoothed to enhance the detection of features at faint levels, are also visible in our [SII] and [OII] images. These clumps of faint emission appear to trace out a fragmented shell, which has possibly undergone Raleigh-Taylor instability (see review of Tenorio-Tagle \& Bodenheimer 1990).

Diffuse ionized gas emission can be seen throughout the disk of the galaxy, however it appears brighter and more structured near concentrations of young, massive stars. Even the more quiescently star-forming outer parts of the galaxy appear to have significant quantities of faint diffuse ionized gas, although in these parts it is largely confined within the plane of the galaxy. The small-scale correlation between diffuse ionized gas and HII regions has been observed in several galaxies (e.g. Walterbos \& Braun 1994, Ferguson et al. 1996) and indeed in NGC 55 (Hoopes et al 1996) and lends support for a model in which the gas is photoionized, either directly or indirectly, by Lyman continuum photons which leak out of individual HII regions. Qualitatively, it also appears that the intensity of local star formation (ie. SFR per unit area) plays a role in determining the surface brightness and the morphology of the surrounding diffuse ionized gas.

There is some evidence for a thick diffuse ionized gas layer in the central regions of NGC 55. Averaged over the central $1.4 \mathrm{kpc}$, the full-width of this layer (in $\mathrm{H} \alpha+[\mathrm{NII}]$ ) at an $\mathrm{EM}=10 \mathrm{pc} \mathrm{cm}{ }^{-6}$ is $\sim 2 \mathrm{kpc}$. This extended emission is also seen in the [OII] and [SII] images, where the layer has a full-width of $\sim 2.6 \mathrm{kpc}$ and $\sim 1.4 \mathrm{kpc}$ respectively, at the aforementioned surface brightness level. Uncertainties in flat-fielding and the possibility of residual instrumental effects make the detection of this layer marginal at present. Future observations will allow us to study this extra-planar ionized gas layer in more detail.

\section{Luminosity of the Ionized Gas}

The global $\mathrm{H} \alpha+[\mathrm{NII}]$ luminosity of NGC 55 is found by summing all pixel values within a large polygonal aperture. We derive a value of $2.0 \times 10^{40} \mathrm{erg} \mathrm{s}^{-1}$; this value is about 
$20 \%$ lower than that recently reported by Hoopes et al (1996). Large scale flat-fielding errors, as well as realistic errors in the continuum scaling factor (estimated to be $\lesssim 3 \%$ ) could produce a systematic uncertainty of $\pm 5-10 \%$ in this measurement. Assuming an IMF and a set of stellar evolutionary tracks, the global $\mathrm{H} \alpha$ luminosity can be translated into a global current star formation rate. Kennicutt, Tamblyn \& Congdon (1995) have calculated the proportionality factor between the two for various IMFs, based on the stellar evolutionary tracks of Schaller et al (1993). Using these calculations for convenience, the global star formation rate of NGC 55, over the mass range $0.1 \mathrm{M}_{\odot} \leq \mathrm{M} \leq 100 \mathrm{M}_{\odot}$, is found to be $0.16 \mathrm{M}_{\odot} \mathrm{yr}^{-1}$, assuming a Salpeter IMF, or $0.15 \mathrm{M}_{\odot} \mathrm{yr}^{-1}$, assuming a modified Miller-Scalo IMF (Kennicutt 1983). These numbers are likely to be upper limits to the true current star formation rate since we expect to have a small contribution from [NII] in our global $\mathrm{H} \alpha$ flux.

Isolating the fraction of the total $\mathrm{H} \alpha$ emission which is produced by diffuse ionized gas is a difficult task, and many different approaches have previously been adopted (e.g. Walterbos \& Braun 1994, Veilleux et al. 1995, Ferguson et al. 1996, Hoopes et al. 1996). In view of the many uncertainties inherent in deriving the diffuse fraction, especially in the case of a highly inclined galaxy (e.g. variable dust extinction in the plane), we choose here to adopt the simplest technique. An isophotal cut in surface brightness was imposed at $\mathrm{EM}=80 \mathrm{pc} \mathrm{cm}^{-6}$, corresponding to the limit below which the $\mathrm{H} \alpha$ emission could largely be classified as filamentary and/or diffuse. We derive a global diffuse fraction in $\mathrm{H} \alpha+[\mathrm{NII}]$ of $19 \% \pm 5 \%$, which can be compared with the value of $\gtrsim 30 \%$ estimated by Hoopes et al. (1996) using a different technique (the uncertainty associated with our measurement is estimated from realistic errors in the large-scale flat-fielding and continuum subtraction). These same authors also present a diffuse fraction measured via an isophotal cut in surface brightness; at our adopted value of $80 \mathrm{pc} \mathrm{cm}^{-6}$, they find a diffuse fraction of $26 \%$, being slightly higher than the value found here.

Luminosities in $\mathrm{H} \alpha+[\mathrm{NII}],[\mathrm{OII}]$ and [SII] were calculated for the central $5 \mathrm{kpc}$ of the galaxy and are $1.3 \times 10^{40} \mathrm{erg} \mathrm{s}^{-1}, 1.2 \times 10^{40} \mathrm{erg} \mathrm{s}^{-1}$ and $3.4 \times 10^{39} \mathrm{erg} \mathrm{s}^{-1}$ respectively, uncorrected for extinction. Systematic uncertainties on these values due to flat-fielding and continuum subtraction errors are estimated to be $\lesssim 10 \%$. We used the $\mathrm{H} \alpha+[\mathrm{NII}]$ image to define a mask in which pixels with $\mathrm{EM} \leq 80 \mathrm{pc} \mathrm{cm}^{-6}$ were replaced with a value of 0 , and those above this limit were replaced with 1 . Multiplying each of the emission-line images by this mask produced images containing predominantly HII region emission. Summing the counts in these images, and subtracting them from the total counts measured on the unmasked images, allowed us to compute the diffuse fractions in the different bands, for the central $5 \mathrm{kpc}$ of the galaxy. Diffuse fractions of $17 \% \pm 3 \%$ were derived in $\mathrm{H} \alpha+[\mathrm{NII}], 29 \%$ $\pm 5 \%$ in [OII] and $26 \% \pm 5 \%$ in [SII]. 
The total and diffuse ionized gas luminosities in each of the emission lines enabled us to calculate the mean line ratios $([\mathrm{OII}] / \mathrm{H} \alpha+[\mathrm{NII}]$ and $[\mathrm{SII}] / \mathrm{H} \alpha+[\mathrm{NII}])$ for the discrete HII regions and the diffuse ionized gas. We find $[\mathrm{OII}] / \mathrm{H} \alpha+[\mathrm{NII}] \sim 0.80$ and $[\mathrm{SII}] / \mathrm{H} \alpha+[\mathrm{NII}] \sim$ 0.24 for the HII regions, and $[\mathrm{OII}] / \mathrm{H} \alpha+[\mathrm{NII}] \sim 1.42$ and $[\mathrm{SII}] / \mathrm{H} \alpha+[\mathrm{NII}] \sim 0.43$ for the diffuse ionized gas. We will investigate this apparent difference in ionization state between the HII regions and the diffuse ionized gas further in the following section.

\section{Line Ratios and Ionization Mechanism of the Gas}

Constraints on the ionization mechanism of the extra-planar gas are provided from our data by the values of the line ratios of $[\mathrm{OII}] / \mathrm{H} \alpha+[\mathrm{NII}]$ and $[\mathrm{SII}] / \mathrm{H} \alpha+[\mathrm{NII}]$. Theories which explain the diffuse ionized gas as being a result of photoionization by a dilute radiation field (e.g. Mathis 1986, Domgörgen \& Mathis 1994) predict these forbidden line ratios should be significantly enhanced in the diffuse ionized gas relative to bright HII regions, and that there should be a smooth trend of increasing ratio with increasing distance from the ionizing source. Predictions from shock-ionization models, particularly for the [OII] line strengths, are rather strongly dependent on parameters such as the shock velocity (Shull \& McKee 1979; Raymond 1980), which may be highly variable, from one location to another.

Our data allow us to study the variation of emission line ratios over wide ranges of surface brightnesses and of morphology, from bright HII region cores to faint DIG features. Small aperture $\left(2^{\prime \prime}\right)$ photometry was carried out at different locations along the features identified in Figures 2 a as well as in the core and halo regions of several bright HII regions. The total flux inside a given aperture was measured separately on each of the $\mathrm{H} \alpha+[\mathrm{NII}]$, $[\mathrm{OII}]$ and $[\mathrm{SII}]$ images and then ratioed. Only those measurements in which the $\mathrm{S} / \mathrm{N} \gtrsim 3$ in both apertures were retained; this eliminated $\sim 10 \%$ of the measurements.

It is important to assess the possible effects of internal extinction on the derived emission line ratios. Internal extinction within NGC 55 should not affect the $[\mathrm{SII}] / \mathrm{H} \alpha+[\mathrm{NII}]$ ratios measured in various features, where the lines are close in wavelength, but may be an important factor in comparing the $[\mathrm{OII}] / \mathrm{H} \alpha+[\mathrm{NII}]$ ratios. The possible amplitude of this may be estimated using the published spectrophotometry of Webster \& Smith (1983), which yields a mean extinction at $\mathrm{H} \beta$ of $\mathrm{C}(\mathrm{H} \beta)=0.35$ for the cores of bright HII regions (ie. $\mathrm{EM} \sim 10^{5} \mathrm{pc} \mathrm{cm}^{-6}$ ) in the disk of the NGC 55. Adopting the LMC reddening law of Howarth (1983), we find that the intrinsic [OII]/H $\alpha$ ratio towards these positions could be a factor of $\sim 1.7$ higher than that observed (this value is only slightly lower if the Milky Way extinction law is adopted instead). If the haloes surrounding the HII regions and the diffuse ionized features located close to the plane of the galaxy were extincted to the same 
extent as the HII region cores, then one would expect a similar effect. We have no direct knowledge the distribution of dust across, or perpendicular to, the disk of NGC 55 however; the two central HII regions studied by Webster \& Smith (1983) show variations in $\mathrm{C}(\mathrm{H} \beta)$ of order 0.2 , indicating that the dust content in these regions is clearly patchy. In view of these uncertainties, we have not attempted to correct our line ratios for internal extinction. The possible influence of dust on the observed measurements should be kept in mind in the following discussion, however it will not change our major conclusions.

\subsection{Trends with $\mathrm{H} \alpha$ Surface Brightness}

Figures $3 \mathrm{a} \& \mathrm{~b}$ show the measured emission line ratios plotted against $\mathrm{H} \alpha$ surface brightness. There is a clear increase in both the $[\mathrm{OII}] / \mathrm{H} \alpha+[\mathrm{NII}]$ and $[\mathrm{SII}] / \mathrm{H} \alpha+[\mathrm{NII}]$ ratios as the $\mathrm{H} \alpha+[\mathrm{NII}]$ surface brightness of the measured feature (within the aperture) decreases. $[\mathrm{OII}] / \mathrm{H} \alpha+[\mathrm{NII}]$ ratios of $0.2-1.2$ are typically found for the HII regions (a mean value of 0.4 is found for the bright cores), whereas the diffuse ionized features have ratios in the range $0.7-2.5$; likewise, $[\mathrm{SII}] / \mathrm{H} \alpha+[\mathrm{NII}]$ ratios of $0.05-0.3$ typify the HII regions (a mean value of 0.08 is observed in the cores) whereas values of $0.2-0.7$ are found in the diffuse gas. Systematic uncertainties in the emission-line ratios due to realistic errors in the continuum subtraction are typically \pm 0.01 for bright HII regions and \pm 0.05 for diffuse ionized features. The mean $[\mathrm{OII}] / \mathrm{H} \alpha+[\mathrm{NII}]$ and $[\mathrm{SII}] / \mathrm{H} \alpha+[\mathrm{NII}]$ ratios measured for the diffuse ionized features are found to be a factor of $\sim 2$ higher than the mean found towards HII regions, and factors of $4-5$ higher than those found in the bright cores of HII regions; this is in agreement with our earlier calculation based on mean luminosities. [SII] $/ \mathrm{H} \alpha$ ratios in this general range have been observed for diffuse ionized gas in the Milky Way and other galaxies and several authors have pointed out the enhancement relative to bright HII regions (e.g. Sivan, Stasinska and Lequeux 1986; Reynolds 1985, Walterbos \& Braun 1994, Hunter 1994; Hoopes et al 1996). Furthermore, Hunter (1984, 1994, 1996) has found enhanced [OII]/[OIII] compared to bright HII regions in many of the ionized filaments and shells in the LMC and other late-type systems.

Figures 3 a \& $\mathrm{b}$ also reveal the presence of a smooth trend in emission line ratio as one proceeds from bright HII region cores, through haloes of HII regions and into the diffuse ionized gas. HII regions and diffuse ionized gas features observed at the same $\mathrm{H} \alpha+[\mathrm{NII}]$ surface brightness have line ratios which are very similar. Such a continuous trend between the faint outskirts of HII regions and diffuse ionized gas was not found in the Milky Way, where a distinct difference between the two is observed to exist (Reynolds 1985, 1988). A very marginal trend in $[\mathrm{SII}] / \mathrm{H} \alpha+[\mathrm{NII}]$ vs $\mathrm{H} \alpha$ surface brightness was seen by Walterbros 

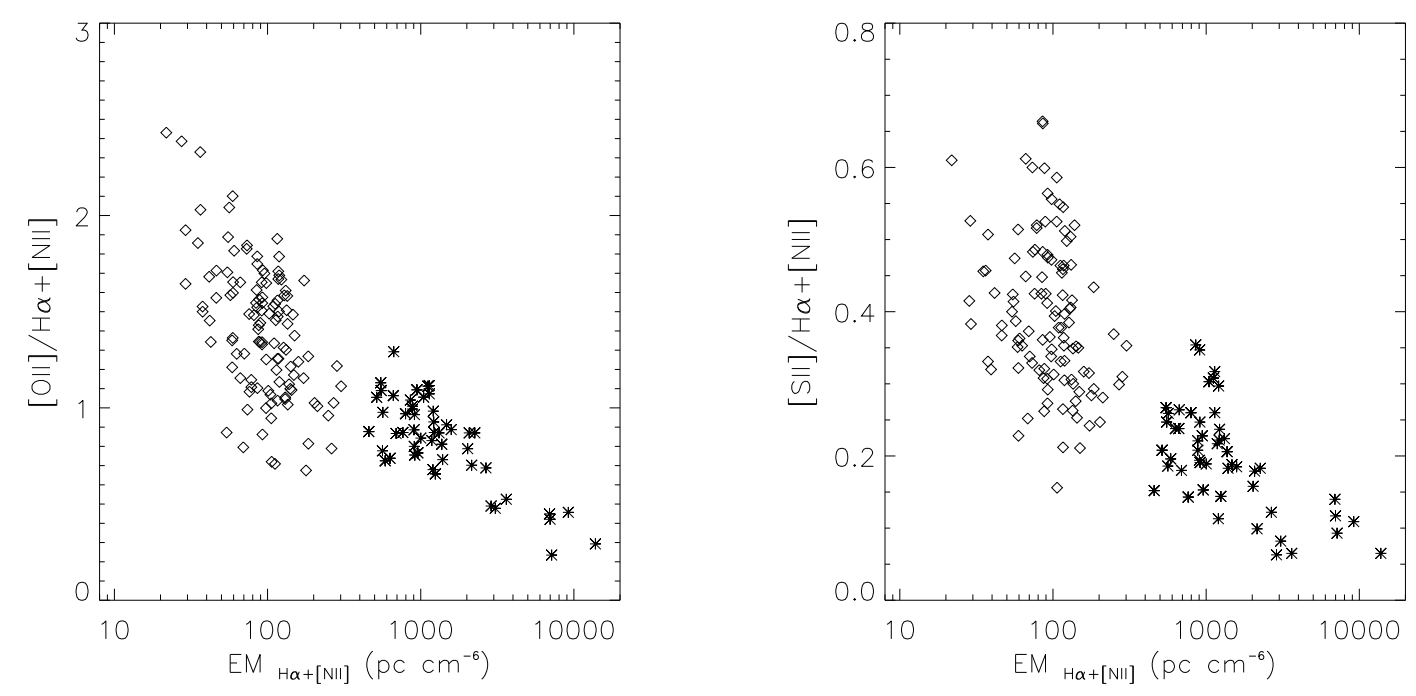

Fig. 3.- (a) The variation of $[\mathrm{OII}] / \mathrm{H} \alpha+[\mathrm{NII}]$ vs $\mathrm{H} \alpha+[\mathrm{NII}]$ surface brightness for various HII regions and diffuse ionized gas features in NGC 55. HII regions are marked by $(*)$ and diffuse ionized gas features by $(\diamond)$. (b) The variation of $[\mathrm{SII}] / \mathrm{H} \alpha+[\mathrm{NII}]$ vs $\mathrm{H} \alpha+[\mathrm{NII}]$ surface brightness for various HII regions and diffuse ionized gas features in NGC 55 .

\& Braun (1994) but was not seen by Hoopes et al (1996) in their study of three Sculptor Group galaxies (including NGC 55). This could be related to the larger apertures these authors used for their aperture photometry, and the lower S/N of their data. Spectroscopic study of several filaments and supershells in the LMC and NGC 1800 by Hunter (1994, 1996) found evidence for a correlation between [OII]/[OIII] and $\mathrm{H} \alpha$ surface brightness, in the sense that lower surface brightness features were characterized by a lower ionization state. The results presented here can be similarily interpreted.

Finally, while some scatter does exist in these plots, different morphological classes of diffuse ionized gas structures (e.g. loops, chimneys, patches of diffuse emission) cannot be distinguished from each other purely on the basis of the line ratios presented here. This has also been observed by Hunter $(1994,1996)$ who studied different classes of ionized structures in the LMC and NGC 1800. This is consistent with all having a common ionization mechanism, which as we discuss in Section 6, is most likely to be photoionization by massive stars. 


\subsection{Trends with Height above the Plane}

Figures $4 \mathrm{a} \& \mathrm{~b}$ show the behavior of the emission line ratios with height above the plane. A clear trend is seen in the $[\mathrm{OII}] / \mathrm{H} \alpha+[\mathrm{NII}]$ ratio, amounting to a factor of $\sim 4$ difference between features in the plane and those above it. The $[\mathrm{SII}] / \mathrm{H} \alpha+[\mathrm{NII}]$ ratio also shows a trend with height above the plane, although there is more scatter. This seems to be due both to low $\mathrm{H} \alpha+[\mathrm{NII}]$ surface brightness features in the plane having a high $[\mathrm{SII}] / \mathrm{H} \alpha+[\mathrm{NII}]$ ratio, as well as some bright extra-planar features which have low $[\mathrm{SII}] / \mathrm{H} \alpha+[\mathrm{NII}]$ ratios. For example, the cap of emission which sits atop feature ' $\mathrm{I}$ ', and the large shell (feature ' $\mathrm{D}$ ') have ionization states more typical of HII regions than diffuse ionized features ('I': $[\mathrm{OII}] / \mathrm{H} \alpha+[\mathrm{NII}] \sim 1,[\mathrm{SII}] / \mathrm{H} \alpha+[\mathrm{NII}] \sim 0.3$, 'D': $[\mathrm{OII}] / \mathrm{H} \alpha+[\mathrm{NII}] \sim 1.5$, $[\mathrm{SII}] / \mathrm{H} \alpha+[\mathrm{NII}] \sim 0.3)$. Graham \& Lawrie (1982) also observed their shell to have unusually strong $[\mathrm{OIII}]$ emission $([\mathrm{OIII}] / \mathrm{H} \beta \sim 1)$. These line ratios could be understood if these structures were being directly ionized by OB associations in the disk below, or by in situ star formation. It should be noted that enhanced $[\mathrm{NII}] / \mathrm{H} \alpha$ ratios have been observed in the extra-planar diffuse ionized gas in NGC 891 (Dettmar \& Schulz 1992) and NGC 4631 (Golla et al 1996); if such an enhancement is also present in the diffuse ionized gas of NGC 55, then this implies that the true $[\mathrm{SII}] / \mathrm{H} \alpha,[\mathrm{OII}] / \mathrm{H} \alpha$ line ratios in the extra-planar gas could be even higher than presented here.
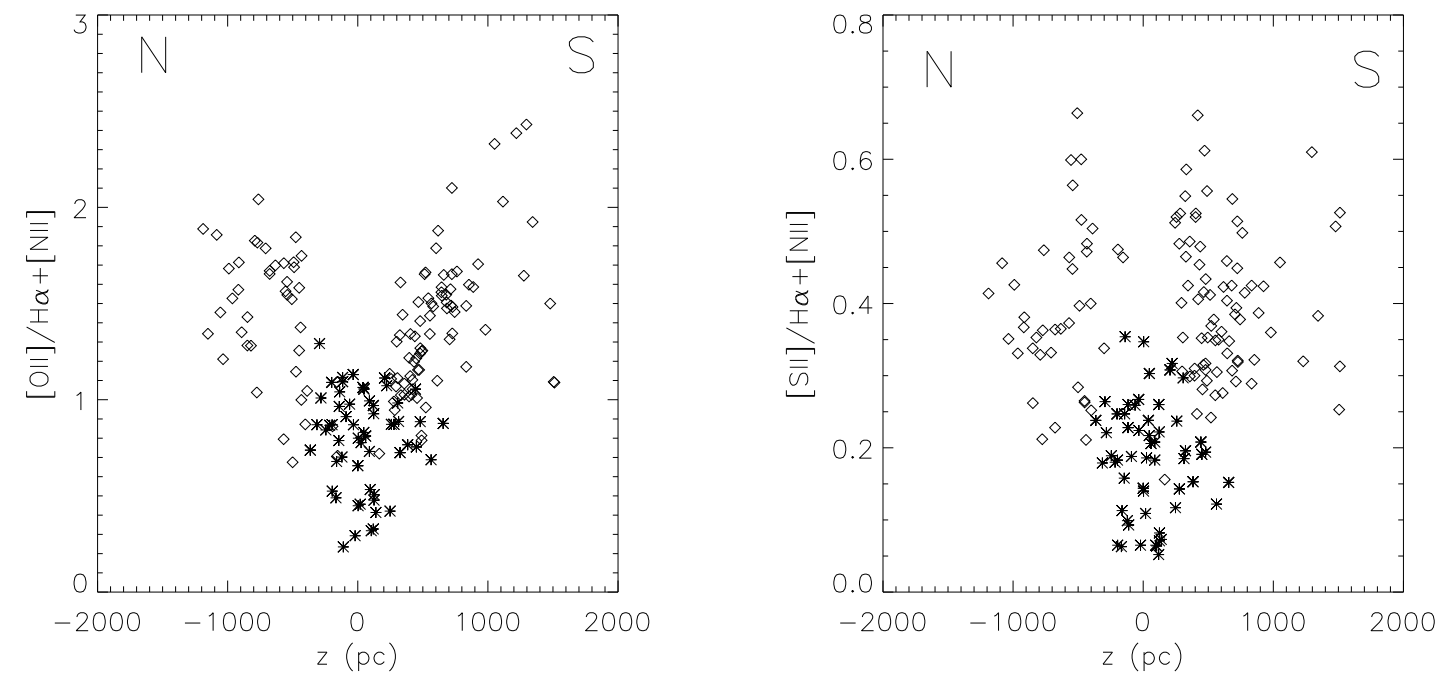

Fig. 4.- (a) The variation of $[\mathrm{OII}] / \mathrm{H} \alpha+[\mathrm{NII}]$ vs $\mathrm{z}$ (height above the plane in parsecs) in NGC 55. HII regions are marked by $(*)$ and diffuse ionized gas features by $(\diamond)$. North and South are labelled. (b) The variation of $[\mathrm{SII}] / \mathrm{H} \alpha+[\mathrm{NII}]$ vs z (height above the plane in parsecs). 
The lack of a well-defined correlation between $[\mathrm{SII}] / \mathrm{H} \alpha+[\mathrm{NII}]$ and $z$ at first appears to be in contrast to the studies of Rand et al (1990), Dettmar \& Schulz (1992) and Golla et al (1996) where a clear trend was seen in the edge-on galaxies NGC 891 and NGC 4631. However, those trends were seen in single vertical cuts taken parallel to the minor axis, intersecting the disk at points of particularly bright HII region emission. Our plots on the other hand show the trend for a variety of disk features and include faint diffuse in disk emission, as well as bright extra-planar filaments and loops. Indeed, experiments with our data have revealed that a single cut through the bright central HII complex shows a significantly stronger correlation between $[\mathrm{SII}] / \mathrm{H} \alpha+[\mathrm{NII}]$ and $z$ than that seen in Figure $4 \mathrm{~b}$.

\section{Discussion}

We have presented a deep $\mathrm{H} \alpha+[\mathrm{NII}],[\mathrm{SII}]$ and [OII] imaging study of the nearby, highly inclined galaxy NGC 55, allowing us to probe the disk-halo interface with unprecedented spatial resolution. NGC 55 hosts a spectacular variety of ionized gas features, protruding typically 1-2 kpc out of the plane of the disk, as well as what appears to be a faint diffuse ionized layer extending over the central regions. The numerous chimneys protruding out of the disk may facilitate the transport of hot gas into the halo and provide low column-density pathways by which UV photons can travel to high scaleheights. A very faint shell of emission is seen which reaches a height of $\sim 2.6 \mathrm{kpc}$.

Despite its low luminosity and current star formation rate, NGC 55 has a disk-halo interface which rivals that seen in galaxies with higher global star formation rates, such NGC 891 and NGC 4631 (Rand 1990; Dettmar 1990). Most of the extra-planar activity seen in NGC 55 is concentrated around the two large HII complexes in the central regions, suggesting that the intensity of local star formation governs both the morphology and the surface brightness of diffuse emission (see also Ferguson et al 1996). Indeed many low mass dwarf and irregular galaxies dominated by a single large HII complex are observed to host a variety of large ionized filamentary structures, some may even protrude out of the plane (e.g. NGC 1800, NGC 1569), despite the fact that their global star formation rates are extremely modest (Marlowe et al 1994; Hunter et al 1993). As expected theoretically, local, rather than global, star formation properties are important for the presence of structured extra-planar ionized gas in disk galaxies. Smoother diffuse ionized gas layers such as seen in the Milky Way and in M31, which lack super-giant HII regions, could require simply a source of ionizing photons, such as leaky HII regions (Leisawitz \& Hauser 1988; De Geus et al 1993) and a relatively porous ISM whereas large extra-planar structures could require more violent star formation, which structures the ISM and results in more pathways along 
which UV photons can travel unscathed. Further observations of edge-on and face-on disk galaxies will allow these ideas to be tested.

One should bear in mind that the means by which gas is physically transported to large distances above the plane may well be different from the process which keeps it ionized. Many factors play a role in the formation of superbubbles and chimneys, including the ambient gas density and magnetic fields (NI89, Tomisaka 1988, Ferriere et al 1989). The strong curvature of many of the filaments towards the plane of the NGC 55 suggests they could shaped by a magnetic field, which is expected to have this geometry if, for example, the Parker instability or related processes are occuring (e.g. Parker 1979). Gas could be clumped along the magnetic field lines and simply illuminated by escaping UV photons, or the magnetic field could be playing a role in confining the expanding structures as they attempt to break out of the disk. The existence of ionized gas at heights of $\sim 2.6 \mathrm{kpc}$ above the plane of the galaxy is particular puzzling. If this gas is photoionized, either directly or indirectly, by photons produced by massive stars in the disk, then extremely long pathlengths through the halo are required.

Our observations have allowed us to demonstrate quantitatively for the first time that there is a continuous trend in $[\mathrm{OII}] / \mathrm{H} \alpha+[\mathrm{NII}]$ and $[\mathrm{SII}] / \mathrm{H} \alpha+[\mathrm{NII}]$ as one proceeds to fainter and fainter surface brightnesses. This trend extends all the way from bright HII region cores to filaments and loops and finally to faint diffuse patches of emission, strongly suggestive that all are ionized by the common process of photoionization. The smooth sequence in ionization state between HII regions and diffuse ionized gas revealed by our plots lends strong support for photoionization of the diffuse gas by a dilute radiation field, such as that produced by massive stars that are located at considerable distances (Mathis 1986, Domgörgen \& Mathis 1994). In such a scenario, one could envisage the Lyman continuum photons escaping from the HII regions where they were produced and travelling significant distances through the ISM. The propagation of this radiation through the disk could be significantly altered by even moderate columns of interstellar material, possibly resulting in a hardened halo radiation field (e.g. Sokolowski 1994). While the smooth sequence in line ratios alone cannot be used to rule out shock ionization of the diffuse gas, the relatively small range in observed values of these features makes a dominant role for shocks rather unattractive, given the dependence on parameters such as shock velocity.

The strong [OII] emission observed above the plane of the galaxy has important implications for the electron temperature, $T_{e}$, of the gas. The [OII] line requires $\sim$ $3.3 \mathrm{eV}$ to be collisionally excited and the intensity of the line is governed by the factor $T_{e}^{-0.5} e^{-3.3 e V / k T_{e}}$ (Spitzer 1978). The temperature dependence of the H $\alpha$ recombination line is approximately $\mathrm{T}_{e}^{-1}$ (Osterbrock 1989). Thus, as $\mathrm{T}_{e}$ decreases from 10,000 K to 5,000K, 
the strength of the $[\mathrm{OII}] / \mathrm{H} \alpha$ line ratio is expected to decrease by a factor of $\sim 65$. Thus, the presence of widespread [OII] emission above the plane of the galaxy requires warm gas in the disk-halo interface. The strength of the [OII] emission also places severe constraints on the power source for the diffuse ionized gas. Studies of the Milky Way and other galaxies have shown that mechanical energy from supernovae and stellar winds can barely satisfy the minimum power requirements for diffuse ionized gas. Strong, ubiquitous [OII] emission implies that the minimum power model is inappropriate for these calculations, suggesting only a minor role for shocks in ionizing the diffuse gas. The observed $[\mathrm{OII}] / \mathrm{H} \alpha+[\mathrm{NII}]$ ratios in the extra-planar ionized features are larger than predicted by the models of Domgörgen and Mathis (1994) for photoionization of gas by a dilute radiation field. This may suggest that a significant hardening of the radiation does indeed take place as photons propagate through the interstellar medium (see Sokolowski 1994). Future spectroscopic observations can extend the sensitivity to ionization states through measurements of the [OIII] line and will also be required to confirm the strength of the $[\mathrm{OII}] / \mathrm{H} \alpha$ emission measurements in this and other galaxies.

The strong [OII] emission from the ionized gas in NGC 55 is of particular interest in the interpretation of the redshifted emission from more distant galaxies. Much of the peculiar structure seen in these galaxies (e.g. Schade et al 1996; Cowie et al 1995) may simply be due to individual star-forming complexes and bright diffuse ionized extensions, such as we have seen here. Indeed, if NGC 55 were placed at $\mathrm{z} \sim 0.6$, it would very likely appear as a 'chain' galaxy in an HST F606W image (Cowie et al 1995). Further [OII] observations of low redshift galaxies, spanning a range of morphological types and star formation rates, will shed more light on the nature and evolutionary state of moderate redshift galaxies.

We thank Tim Heckman, Piero Rosati and Deidre Hunter for stimulating discussions and Don Cox for organizing an excellent Diffuse Interstellar Medium session at the Madison, AAS meeting where we presented preliminary results and enjoyed many discussions with participants. We thank the staff of Cerro Tololo Inter-American Observatory for their excellent support. AMNF acknowledges support from the Zonta International Foundation in the form of an Amelia Earhart Fellowship. This research has been supported in part by NASA grant NAGW-2892. 


\section{REFERENCES}

Bergeron, J. \& Boisse, P. 1991, A\&A, 243, 344

Bloemen, H. 1990, IAU Symp. 144, The Interstellar Disk-Halo Connection in Galaxies (Dordrecht: Kluwer)

Bomans, D. J. \& Grebel, E. K. 1993, in Violent Star Formation, edited by G. Tenorio-Tagle (Cambridge University Press, Cambridge), p. 156

Cowie, L. L., Hu, E. M. \& Songaila, A. 1995, AJ, 110, 1526

Davies, R. D., Elliot, K. J. \& Meaburn, J. 1976, MNRAS, 81, 89

De Geus, E. J., Vogel, S. N., Digel, S. W. \& Gruendl, R. A. 1993, ApJ, 413, L97

de Vaucouleurs, G., de Vaucouleurs, A., Corwin, H. R., Buta, R. J., Paturel, G., \& Fouquè, P. 1991, Third Reference Catalogue of Bright Galaxies (Springer-Verlag, New York) (RC3)

Dettmar, R. J. 1990, A\&A, 232, L15

Dettmar, R. J. \& Schulz, H. 1992, A\&A, 254, 25

Domgörgen, H. \& Mathis, J. S. 1994, ApJ, 428, 647

Ferguson, A. M. N., Wyse, R. F. G., Gallagher, J. S. \& Hunter, D. A. 1996, AJ, 111, 2265

Ferriere, K., MacLow, M.-M. \& Zweibel, E. G. 1991, ApJ, 375, 239

Golla, G., Dettmar, R.-J. \& Domgörgen, H. 1996, preprint

Graham, J. A. \& Lawrie, D. G. 1982, ApJ, 253, L73

Heiles, C. 1984, ApJS, 55, 585

Heiles, C. 1990, ApJ, 354, 483

Hester, J. J. \& Kulkarni, S. R. 1990, in The Interstellar Medium in External Galaxies, edited by D. Hollenbach \& H. Thronson (NASA CP-3084), p. 288

Hoopes, C. G., Walterbos, R. A. M. \& Greenawalt, B. E. 1996, astro-ph/9607048

Howarth, I. 1983, MNRAS, 203, 301

Hunter, D. A. 1984, ApJ, 276, L35

Hunter, D. A. \& Gallagher, J. S. 1992, ApJ, 391, L9

Hunter, D. A. \& Gallagher, J. S. 1997, ApJ, in press

Hunter, D. A., Hawley, W. N. \& Gallagher, J. S. 1993, AJ, 106, 1797

Hunter, D. A. 1994, AJ, 107, 565 
Hunter, D. A. 1996, ApJ, 457, 671

Kennicutt, R. C. 1983, ApJ, 272, 54

Kennicutt, R. C. 1984, ApJ, 287, 116

Kennicutt, R. C. 1988, ApJ, 334, 144

Kennicutt, R. C., Tamblyn, P. \& Congdon, C. E. 1994, ApJ, 435,22

Kennicutt, R. C., Bresolin, F. , Bomans, D. J., Bothun, G. D. \& Thompson, I. B. 1995, AJ, 109, 594

Kulkarni, S. R. \& Heiles, C. 1988, in Galactic and Extragalactic Radio Astronomy, edited by G. Verschuur \& K. I. Kellerman (Springer, New York), p. 95

Leisawitz, D. \& Hauser, M. G. 1988, ApJ, 332, L954

MacLow, M.-M., McCray, R. \& Norman, M. L. 1988, ApJ, 337, 141

Marlowe, A. T., Heckman, T. M., Wyse, R. F. G. \& Schommer, R. 1995, ApJ, 438, 563

Mathis, J. S. 1986, ApJ, 301, 423

Monnet, G. 1971, A\&A, 12, 379

Norman, C. A. \& Ikeuchi, I. 1989,ApJ, 345, 372

Normandeau, M., Taylor, A. R. \& Dewdney, P. E. 1996, Nature, 380, 687

Osterbrock, D. E. 1989, Astrophysics of Gaseous Nebulae and Active Galactic Nuclei (Mill Valley: University Science Books)

Parker, E. 1979, Cosmic Magnetic Fields: Their Origin and Their Activity (Oxford:Clarendon)

Pildis, R. A., Bregman, J. N. \& Schombert, J. M. 1994, ApJ, 427, 160

Puche, D., Carignan, C. \& Wainscoat, R. J. 1991, AJ, 101, 447

Rand, R. J., Kulkarni, S. R. \& Hester, J. J. 1990, ApJ, 352, L1

Rand, R. J., Kulkarni, S. R. \& Hester, J. J. 1992, ApJ, 396, 97

Rand, R. J. 1996, ApJ, 463, 712

Raymond, J. C. 1979, ApJS, 39, 1

Raymond, J. C. 1992, ApJ, 384, 502

Reynolds, R. J., Roesler, F. L., Scherb, F., \& Boldt, E. 1971, in The Gum Nebula and Related Problems, edited by S. P. Maran, J. C. Brandt \& T. P. Stecher (NASA SP-332), p. 169

Reynolds, R. J. 1984, ApJ, 282, 193 
Reynolds, R. J. 1985, ApJ, 294, 256

Reynolds, R. J. 1988, ApJ, 333, 341

Schade, D., Lilly, S. J., Crampton, D., Hammer, F., Le Fevre, O., \& Tresse, L. 1995, /apj, 451, L1

Scaller, G., Schaerer, D., Meynet, G. \& Maeder, A. 1993, A\&AS, 96, 269

Shapiro, P. R. \& Field, G. B. 1976, ApJ, 205, 762

Shull, J. M. \& McKee, C. F. 1979, ApJ, 227, 131

Sivan, J.-P., Stasinska, G. \& Lequeux, J. 1986, A\&A, 158, 279

Slavin, J. D., Shull, J. M. \& Begelman, M. C. 1993, ApJ, 407, 83

Sokolowski, J. 1993, preprint

Spitzer, L., Jr. 1978, Physical Processes in the Interstellar Medium (Wiley: New York)

Stone, R. P. S. \& Baldwin, J. A. 1983, MNRAS, 204, 347

Tomisaka, K. 1990, ApJ, 361, L5

Tenorio-Tagle, G. \& Bodenheimer, P. 1988, ARA\&A, 26, 145

Veilleux, S., Cecil, G. \& Bland-Hawthorn, J. 1995, ApJ, 445, 152

Walterbos, R. A. M. \& Braun, R. 1994, ApJ, 431, 156 (WB94)

Webster, B. L. \& Smith, M. G. 1983, MNRAS, 204, 743 


\section{FIGURES}

Figure 1. A mosaiced continuum-subtracted $\mathrm{H} \alpha$ image of NGC 55 displayed with a logarithmic stretch. The image shows the galaxy out to $\sim R_{25}$. North is up and east is the left.

Figure 2(a). A continuum-subtracted $\mathrm{H} \alpha$ image of the central $5 \mathrm{kpc}$ of NGC 55, shown on a linear scale with individual features marked. (b) has been smoothed with a gaussian with $\mathrm{FWHM}=2.5^{\prime \prime}$ to enhance the low surface brightness features.

Figure 3(a). The variation of $[\mathrm{OII}] / \mathrm{H} \alpha+[\mathrm{NII}]$ vs $\mathrm{H} \alpha+[\mathrm{NII}]$ surface brightness for various HII regions and diffuse ionized gas features in NGC 55. HII regions are marked by $(*)$ and diffuse ionized gas features by $(\diamond)$. (b) The variation of $[\mathrm{SII}] / \mathrm{H} \alpha+[\mathrm{NII}]$ vs $\mathrm{H} \alpha+[\mathrm{NII}]$ surface brightness for various HII regions and diffuse ionized gas features in NGC 55 .

Figure 4(a) The variation of $[\mathrm{OII}] / \mathrm{H} \alpha+[\mathrm{NII}]$ vs $\mathrm{z}$ (height above the plane in parsecs) in NGC 55. HII regions are marked by $(*)$ and diffuse ionized gas features by $(\diamond)$. North and South are labelled. (b) The variation of $[\mathrm{SII}] / \mathrm{H} \alpha+[\mathrm{NII}]$ vs $\mathrm{z}$ (height above the plane in parsecs). 


\section{Table 1. Basic Properties of NGC 55}

\begin{tabular}{ccc}
\hline \hline & & \\
& & References \\
\hline R. A. (2000.0) & $00^{h} 15^{m} 08.5^{s}$ & 1 \\
Dec. (2000.0) & $-39^{\circ} 13^{\prime} 13.3^{\prime \prime}$ & 1 \\
Morphological Type & $\mathrm{SB}(\mathrm{s}) \mathrm{m}$ & 1 \\
Distance (Mpc) & $1.60 \mathrm{Mpc}$ & 2 \\
$\mathrm{~V}_{\text {helio }}$ & $118 \mathrm{~km} / \mathrm{s}$ & 2 \\
$i$ & $79^{\circ}$ & 2 \\
$\mathrm{R}_{25}$ & $18.9^{\prime}=8.8 \mathrm{kpc}$ & 2 \\
$\mathrm{R}_{\text {Holm }}$ & $20.2^{\prime}=9.5 \mathrm{kpc}$ & 2 \\
$\mathrm{M}_{B}^{0}$ & -18.4 & 1 \\
\hline \hline
\end{tabular}

References for Table 1.

(1) RC3; (2) Puche, Carignan \& Wainscoat (1991) 
TABle 2. Properties of Various Ionized Features

\begin{tabular}{|c|c|c|c|c|}
\hline Feature & Description & Dimensions & Max. Height Above Plane & $\mathrm{H} \alpha+[\mathrm{NII}]$ Surface Brightness \\
\hline $\mathrm{B}$ & chimney & width $\sim 50 \mathrm{pc}$ & $400 \mathrm{pc}$ & $23-60$ \\
\hline $\mathrm{D}$ & loop & diam $\sim 815 \mathrm{pc}$, width $\sim 30 \mathrm{pc}$ & $960 \mathrm{pc}$ & $20-40$ \\
\hline $\mathrm{E}$ & chimney & width $\sim 60 \mathrm{pc}$ & $1.3 \mathrm{kpc}$ & $10-30$ \\
\hline $\mathrm{F}$ & complex of filaments & $800 \mathrm{pc} \times 800 \mathrm{pc}$ & $1.6 \mathrm{kpc}$ & $30-60$ \\
\hline G & chimney & width $\sim 100 \mathrm{pc}$ & $1.5 \mathrm{kpc}$ & $15-20$ \\
\hline $\mathrm{J}$ & diffuse disk emission & $\cdots$ & $\cdots$ & $20-50$ \\
\hline $\mathrm{K}$ & chimney & $\cdots$ & $1.3 \mathrm{kpc}$ & 15 \\
\hline $\mathrm{L}$ & bubble & diam $\sim 160 \mathrm{pc}$, width $\sim 30 \mathrm{pc}$ & $\cdots$ & rim $\sim 60-120$, inside $\sim 20-30$ \\
\hline $\mathrm{M}$ & loop? & diam $\sim 1.6 \mathrm{kpc}$ & $2.6 \mathrm{kpc}$ & $\lesssim 5$ \\
\hline
\end{tabular}

${ }^{1}$ measured in EM, assuming $\mathrm{T}_{e}=10^{4} \mathrm{~K}$ 